\title{
WEIGHTED PSEUDO-ALMOST PERIODIC SOLUTIONS FOR SOME ABSTRACT DIFFERENTIAL EQUATIONS WITH UNIFORM CONTINUITY
}

\author{
LI-LI ZHANG and HONG-XU LI ${ }^{凶}$
}

(Received 16 April 2009)

\begin{abstract}
In this work, we give some theorems on (mild) weighted pseudo-almost periodic solutions for some abstract semilinear differential equations with uniform continuity. To facilitate this we give a new composition theorem of weighted pseudo-almost periodic functions. Our composition theorem improves the known one by making use of a uniform continuity condition instead of the Lipschitz condition.
\end{abstract}

2000 Mathematics subject classification: primary 34C27; secondary 34G20, 42A75.

Keywords and phrases: almost periodic, weighted pseudo-almost periodic, uniform continuity, fixed point, $C_{0}$-semigroup.

\section{Introduction}

The qualitative theory of differential equations involving almost periodicity has been an attractive topic for nearly a century because of their significance and applications in areas such as physics, mathematical biology, and control theory. As a result, several concepts were introduced as generalizations or restrictions of almost periodicity, such as asymptotic almost periodicity, almost automorphism, and pseudo-almost periodicity (see, for example, $[5,11,13-16])$. Consequently, differential equations, partial differential equations, and functional differential equations with these properties have been of great interest to many authors and there is a vast literature on the subject (see, for example, $[1-7,9,11-16]$ and the references therein).

Recently, Diagana $[8,10]$ introduced a new class of functions called weighted pseudo-almost periodic functions, which are a natural generalization of the classical pseudo-almost periodic functions, and discussed the properties of this new class of functions, including a composition result. As applications, some existence and uniqueness theorems for weighted pseudo-almost periodic solutions for abstract differential equations were obtained. We notice that a Lipschitz condition is needed in the composition theorem and its applications in abstract differential equations (see [10, Theorems 3.7, 4.2]). So it is interesting and worthwhile to consider the same problem

(C) 2010 Australian Mathematical Publishing Association Inc. 0004-9727/2010 \$16.00 
under a uniform continuity condition instead of the Lipschitz condition. This seems reasonable and necessary since the uniform continuity condition is the main condition needed for the composition theorems of almost periodic functions and pseudo-almost periodic functions (see $[11,12]$ ).

The aim of this paper is to give some theorems on (mild) weighted pseudo-almost periodic solutions of the abstract semilinear differential equation

$$
u^{\prime}(t)=A u(t)+f(t, u(t)), \quad t \in \mathbb{R},
$$

under a uniform continuity condition. For this purpose, we give a new composition theorem for weighted pseudo-almost periodic functions, which improves the one given in [10] by making use of a uniform continuity condition instead of the Lipschitz condition (see Remark 3.3).

The paper is organized as follows. Some notation and preliminary results are presented in Section 2. A composition theorem for weighted pseudo-almost periodic functions under a uniform continuity condition is proved in Section 3. Then, in Section 4, an existence theorem of mild weighted pseudo-almost periodic solutions for the abstract semilinear differential equation (1.1) is obtained by making use of Schauder's fixed point theorem and the composition theorem obtained in the previous section. Moreover, a theorem on the weighted pseudo-almost periodic solutions for (1.1) is presented. At the end of this paper, an example involving the heat equation is given, where the uniform continuity condition is satisfied and the Lipschitz condition is not satisfied.

\section{Preliminaries}

Throughout this paper, we assume that $(\mathbb{X},\|\cdot\|),(\mathbb{Y},\|\cdot\|)$ are Banach spaces. Let $B C(\mathbb{R}, \mathbb{X})$ (respectively, $B C(\mathbb{R} \times \mathbb{Y}, \mathbb{X})$ ) be the space of bounded continuous functions $u: \mathbb{R} \rightarrow \mathbb{X}$ (respectively, $u: \mathbb{R} \times \mathbb{Y} \rightarrow \mathbb{X}$ ). $B C(\mathbb{R}, \mathbb{X})$ equipped with the sup norm defined by

$$
\|u\|=\sup _{t \in \mathbb{R}}\|u(t)\|
$$

is a Banach space. Furthermore, $C(\mathbb{R}, \mathbb{X})$ (respectively, $C(\mathbb{R} \times \mathbb{Y}, \mathbb{X})$ ) denotes the space of continuous functions from $\mathbb{R}$ to $\mathbb{X}$ (respectively, from $\mathbb{R} \times \mathbb{Y}$ to $\mathbb{X}$ ).

Let $U$ denote the collection of functions (weights) $\rho: \mathbb{R} \rightarrow(0,+\infty)$, which are locally integrable over $\mathbb{R}$. If $\rho \in U$ and for $T>0$, we then set

$$
\mu(T, \rho):=\int_{-T}^{T} \rho(t) d t .
$$

Denote

$$
U_{\infty}:=\left\{\rho \in U: \lim _{T \rightarrow \infty} \mu(T, \rho)=\infty\right\}
$$

and

$$
U_{B}:=\left\{\rho \in U_{\infty}: \rho \text { is bounded with } \inf _{x \in \mathbb{R}} \rho(x)>0\right\} .
$$

Obviously, $U_{B} \subset U_{\infty} \subset U$, with strict inclusions. 
Let $\rho_{1}, \rho_{2} \in U_{\infty}$. Then $\rho_{1}$ is said to be equivalent to $\rho_{2}$, that is, $\rho_{1} \prec \rho_{2}$, if $\rho_{1} / \rho_{2} \in U_{B}$. Then ' $\prec$ ' is a binary equivalence relation on $U_{\infty}$ (see [10]). Let $\rho \in U_{\infty}$, $s \in \mathbb{R}$, and define $\rho_{s}$ by $\rho_{s}(t)=\rho(t+s)$ for $t \in \mathbb{R}$. We denote

$$
U_{T}=\left\{\rho \in U_{\infty}: \rho \prec \rho_{s} \text { for each } s \in \mathbb{R}\right\} .
$$

It is easy to see that $U_{T}$ contains plenty of weights-for example, $1,\left(2+t^{2}\right) /\left(1+t^{2}\right)$, $e^{t}$, and $1+|t|^{n}$ with $n \in \mathbb{N}$.

\section{DEFINITION 2.1.}

(i) A function $f \in C(\mathbb{R}, \mathbb{X})$ is said to be almost periodic if for each $\varepsilon>0$ there exists $\ell(\varepsilon)>0$ such that every interval of length $\ell(\varepsilon)$ contains a number $\tau$ with the property that $\|f(t+\tau)-f(t)\|<\varepsilon$ for each $t \in \mathbb{R}$. Denote by $A P(\mathbb{X})$ the set of all such functions.

(ii) A function $F \in C(\mathbb{R} \times \mathbb{Y}, \mathbb{X})$ is said to be almost periodic in $t \in \mathbb{R}$ uniformly in $y \in \mathbb{Y}$ if for each $\varepsilon>0$ and any compact set $K \subset \mathbb{Y}$ there exists $\ell(\varepsilon)$ such that every interval of length $\ell(\varepsilon)$ contains a number $\tau$ with the property that $\|F(t+\tau, y)-F(t, y)\|<\varepsilon$ for each $t \in \mathbb{R}, y \in \mathbb{Y}$. Denote by $A P(\mathbb{Y}, \mathbb{X})$ the set of all such functions.

For $\rho \in U_{\infty}$, the weighted ergodic space $P A P_{0}(\mathbb{X}, \rho)$ and $P A P_{0}(\mathbb{Y}, \mathbb{X}, \rho)$ are defined by

$$
\begin{aligned}
& \operatorname{PAP}_{0}(\mathbb{X}, \rho):=\left\{f \in B C(\mathbb{R}, \mathbb{X}): \lim _{T \rightarrow \infty} \frac{1}{\mu(T, \rho)} \int_{-T}^{T}\|f(t)\| \rho(t) d t=0\right\} \\
& \operatorname{PAP}_{0}(\mathbb{Y}, \mathbb{X}, \rho) \\
& \quad:=\left\{\begin{array}{c}
F \in B C(\mathbb{R} \times \mathbb{Y}, \mathbb{X}): F(\cdot, y) \text { is bounded for each } y \in \mathbb{Y} \text { and } \\
\lim _{T \rightarrow \infty} \frac{1}{\mu(T, \rho)} \int_{-T}^{T}\|F(t, y)\| \rho(t) d t=0 \text { uniformly in } y \in \mathbb{Y}
\end{array}\right\} .
\end{aligned}
$$

\section{DEFINITION 2.2 [10].}

(i) Let $\rho \in U_{\infty}$. A function $f \in B C(\mathbb{R}, \mathbb{X})$ is called weighted pseudo-almost periodic (or $\rho$-pseudo-almost periodic) if it can be expressed as $f=g+\phi$, where $g \in A P(\mathbb{X})$ and $\phi \in P A P_{0}(\mathbb{X}, \rho)$. Denote by $P A P(\mathbb{X}, \rho)$ the set of all such functions.

(ii) Let $\rho \in U_{\infty}$. A function $F \in C(\mathbb{R} \times \mathbb{Y}, \mathbb{X})$ is called weighted pseudo-almost periodic (or $\rho$-pseudo-almost periodic) in $t \in \mathbb{R}$ and uniformly in $y \in \mathbb{Y}$ if it can be expressed as $F=G+\Phi$, where $G \in A P(\mathbb{Y}, \mathbb{X})$ and $\Phi \in P A P_{0}(\mathbb{Y}, \mathbb{X}, \rho)$. Denote by $P A P(\mathbb{Y}, \mathbb{X}, \rho)$ the set of all such functions.

The functions $g$ and $\phi$ (or $G$ and $\Phi$ ) in Definition 2.2 are called the almost periodic and the weighted ergodic perturbation components of $f$ (or $F$ ), respectively. Moreover, the decomposition $g+\phi$ of $f$ (or $G+\Phi$ of $F$ ) is unique, and $P A P_{0}(\mathbb{X}, \rho$ ) and $P A P(\mathbb{X}, \rho)$ are all Banach spaces with the norm inherited from $B C(\mathbb{R}, \mathbb{X})$ (see [10]). 


\section{Composition theorem}

In this section, we give a composition theorem for weighted pseudo-almost periodic functions under the following uniform continuity condition for $f \in P A P(\mathbb{Y}, \mathbb{X}, \rho)$ with $\rho \in U_{\infty}$ :

$\left(\mathrm{H}_{1}\right) f(t, \cdot)$ is uniformly continuous in each bounded subset of $\mathbb{Y}$ uniformly in $t \in \mathbb{R}$. More explicitly, given $\varepsilon>0$ and $K \subset \mathbb{Y}$ bounded, there exists $\delta>0$ such that $x, y \in K$ and $\|x-y\|<\delta$ imply that $\|f(t, x)-f(t, y)\|<\varepsilon$ for all $t \in \mathbb{R}$.

The following lemma will be used in the proof of the composition theorem.

LEMMA 3.1. Let $\rho \in U_{\infty}$ and $f \in P A P_{0}(\mathbb{X}, \rho)$. Then, given $\varepsilon>0$,

$$
\lim _{T \rightarrow \infty} \frac{1}{\mu(T, \rho)} \int_{M(T, \varepsilon, f)} \rho(t) d t=0,
$$

where $M(T, \varepsilon, f)=\{t \in[-T, T]:\|f(t)\| \geq \varepsilon\}$.

PROOF. Suppose, on the contrary, that there exists $\varepsilon_{0}>0$ such that

$$
\frac{1}{\mu(T, \rho)} \int_{M\left(T, \varepsilon_{0}, f\right)} \rho(t) d t
$$

does not converge to 0 as $T \rightarrow \infty$. Since $\rho$ is positive, there exists $\delta>0$ such that for each $n$,

$$
\frac{1}{\mu\left(T_{n}, \rho\right)} \int_{M\left(T_{n}, \varepsilon_{0}, f\right)} \rho(t) d t \geq \delta \quad \text { for some } T_{n} \geq n
$$

Then

$$
\begin{aligned}
\frac{1}{\mu\left(T_{n}, \rho\right)} \int_{-T_{n}}^{T_{n}}\|f(t)\| \rho(t) d t & \geq \frac{1}{\mu\left(T_{n}, \rho\right)} \int_{M\left(T_{n}, \varepsilon_{0}, f\right)}\|f(t)\| \rho(t) d t \\
& \geq \frac{\varepsilon_{0}}{\mu\left(T_{n}, \rho\right)} \int_{M\left(T_{n}, \varepsilon_{0}, f\right)} \rho(t) d t \\
& \geq \varepsilon_{0} \delta
\end{aligned}
$$

which contradicts the fact that $f \in P A P_{0}(\mathbb{X}, \rho)$, and the proof is complete.

We are now ready to give the composition theorem of weighted pseudo-almost periodic functions.

THEOREM 3.2. Let $f \in P A P(\mathbb{Y}, \mathbb{X}, \rho)$ and $h \in P A P(\mathbb{Y}, \rho)$ with $\rho \in U_{\infty}$. Assume that condition $\left(H_{1}\right)$ and the following condition hold:

$\left(\mathrm{H}_{2}\right) f(\mathbb{R}, K)=\{f(t, x): t \in \mathbb{R}, x \in K\}$ is bounded for every bounded subset $K \subset \mathbb{Y}$.

Then $f(\cdot, h(\cdot)) \in \operatorname{PAP}(\mathbb{X}, \rho)$ with almost periodic component $f_{\mathrm{ap}}\left(\cdot, h_{\mathrm{ap}}(\cdot)\right)$, where $f_{\mathrm{ap}}$ and $h_{\mathrm{ap}}$ are the almost periodic components of $f$ and $h$, respectively. 
Proof. Since $h \in P A P(\mathbb{Y}, \rho) \subset B C(\mathbb{R}, \mathbb{Y})$, we know that $f(\cdot, h(\cdot)) \in B C(\mathbb{R}, \mathbb{X})$. Let $f=f_{\text {ap }}+f_{e}$ and $h=h_{\text {ap }}+h_{e}$ with $f_{\text {ap }} \in A P(\mathbb{Y}, \mathbb{X}), f_{e} \in P A P_{0}(\mathbb{Y}, \mathbb{X}, \rho)$, $h_{\text {ap }} \in A P(\mathbb{Y})$ and $h_{e} \in P A P_{0}(\mathbb{Y}, \rho)$. Then

$$
f(t, h(t))=f_{\text {ap }}\left(t, h_{\text {ap }}(t)\right)+f(t, h(t))-f\left(t, h_{\text {ap }}(t)\right)+f_{e}\left(t, h_{\text {ap }}(t)\right) .
$$

It is clear that $f_{\text {ap }}\left(\cdot, h_{\text {ap }}(\cdot)\right) \in A P(\mathbb{X})$ (see [11, Theorem 1.9]). So we only need to show that

$$
f(\cdot, h(\cdot))-f\left(\cdot, h_{\text {ap }}(\cdot)\right)+f_{e}\left(\cdot, h_{\text {ap }}(\cdot)\right) \in P A P_{0}(\mathbb{X}, \rho) .
$$

This will be done in the following two steps.

Step 1 . We prove that $f(\cdot, h(\cdot))-f\left(\cdot, h_{\mathrm{ap}}(\cdot)\right) \in P A P_{0}(\mathbb{X}, \rho)$.

Let $K \subset \mathbb{Y}$ be bounded such that $h(\mathbb{R}), h_{\text {ap }}(\mathbb{R}) \subset K$. Then, by assumption $\left(\mathrm{H}_{2}\right)$, there exists $S>0$ such that

$$
\left\|f(t, h(t))-f\left(t, h_{\mathrm{ap}}(t)\right)\right\| \leq S \quad \text { for all } t \in \mathbb{R} .
$$

Meanwhile, by condition $\left(\mathrm{H}_{1}\right)$, given $\varepsilon>0$, there exists $\delta>0$, such that for $x, y \in K$ with $\|x-y\|<\delta$,

$$
\|f(t, x)-f(t, y)\|<\frac{\varepsilon}{2} \quad \text { for all } t \in \mathbb{R} .
$$

It follows from Lemma 3.1 that

$$
\lim _{T \rightarrow \infty} \frac{1}{\mu(T, \rho)} \int_{M\left(T, \delta, h_{e}\right)} \rho(t) d t=0,
$$

where $M\left(T, \delta, h_{e}\right)=\left\{t \in[-T, T]:\left\|h_{e}(t)\right\| \geq \delta\right\}$. Thus, there exists $T_{0}>0$ such that

$$
\frac{1}{\mu(T, \rho)} \int_{M\left(T, \delta, h_{e}\right)} \rho(t) d t<\frac{\varepsilon}{2 S} \quad \text { for all } T>T_{0} .
$$

Noticing that

$$
\left\|h(t)-h_{\mathrm{ap}}(t)\right\|=\left\|h_{e}(t)\right\|<\delta, \quad \text { for all } t \in[-T, T] \backslash M\left(T, \delta, h_{e}\right),
$$

by (3.1)-(3.3) we have, for $T>T_{0}$,

$$
\begin{aligned}
\frac{1}{\mu(T, \rho)} & \int_{-T}^{T}\left\|f(t, h(t))-f\left(t, h_{\mathrm{ap}}(t)\right)\right\| \rho(t) d t \\
= & \frac{1}{\mu(T, \rho)} \int_{M\left(T, \delta, h_{e}\right)}\left\|f(t, h(t))-f\left(t, h_{\mathrm{ap}}(t)\right)\right\| \rho(t) d t \\
& +\frac{1}{\mu(T, \rho)} \int_{[-T, T] \backslash M\left(T, \delta, h_{e}\right)}\left\|f(t, h(t))-f\left(t, h_{\mathrm{ap}}(t)\right)\right\| \rho(t) d t \\
\leq & \frac{S}{\mu(T, \rho)} \int_{M\left(T, \delta, h_{e}\right)} \rho(t) d t+\frac{\varepsilon}{2 \mu(T, \rho)} \int_{[-T, T] \backslash M\left(T, \delta, h_{e}\right)} \rho(t) d t \\
< & S \frac{\varepsilon}{2 S}+\frac{\varepsilon}{2}=\varepsilon .
\end{aligned}
$$

This implies the conclusion. 
Step 2. We prove that $f_{e}\left(\cdot, h_{\text {ap }}(\cdot)\right) \in P A P_{0}(\mathbb{X}, \rho)$.

Since $h_{\text {ap }} \in A P(\mathbb{Y})$ and $f_{\text {ap }} \in A P(\mathbb{Y}, \mathbb{X}), \overline{h_{\text {ap }}(\mathbb{R})}$ is compact and $f_{\text {ap }}$ is uniformly continuous in $\mathbb{R} \times \overline{h_{\mathrm{ap}}(\mathbb{R})}$. Then it follows from condition $\left(\mathrm{H}_{1}\right)$ that $f_{e}(t, x)=$ $f(t, x)-f_{\mathrm{ap}}(t, x)$ is uniformly continuous in $x \in \overline{h_{\mathrm{ap}}(\mathbb{R})}$ uniformly in $t$. That is, given $\varepsilon>0$, there exists $\delta>0$ such that, for $x, y \in \overline{h_{\text {ap }}(\mathbb{R})}$ with $\|x-y\|<\delta$,

$$
\left\|f_{e}(t, x)-f_{e}(t, y)\right\|<\frac{\varepsilon}{2} \quad \text { for all } t \in \mathbb{R} .
$$

Meanwhile, one can find in $h_{\text {ap }}(\mathbb{R})$ a finite $\delta$-net of $\overline{h_{\mathrm{ap}}(\mathbb{R})}$. Namely, there exist a finite number of points $x_{1}, x_{2}, \ldots, x_{m} \in h_{\mathrm{ap}}(\mathbb{R})$ such that, for any $y \in \overline{h_{\mathrm{ap}}(\mathbb{R})}$, we have $\left\|y-x_{k}\right\|<\delta$ for some $1 \leq k \leq m$. Let

$$
\mathcal{O}_{k}=\left\{t \in \mathbb{R}:\left\|h_{\mathrm{ap}}(t)-x_{k}\right\|<\delta\right\}, \quad k=1,2, \ldots, m .
$$

Then $\mathbb{R}=\bigcup_{k=1}^{m} \mathcal{O}_{k}$. Let

$$
\mathcal{B}_{1}=\mathcal{O}_{1}, \quad \mathcal{B}_{k}=\mathcal{O}_{k} \backslash\left(\bigcup_{i=1}^{k-1} \mathcal{O}_{i}\right), \quad k=2,3, \ldots, m .
$$

Then

$$
\mathbb{R}=\bigcup_{k=1}^{m} \mathcal{B}_{k} \quad \text { and } \quad \mathcal{B}_{j} \cap \mathcal{B}_{k}=\emptyset, \quad j \neq k
$$

Moreover, by (3.4)

$$
\left\|f_{e}\left(t, h_{\text {ap }}(t)\right)-f_{e}\left(t, x_{k}\right)\right\|<\frac{\varepsilon}{2} \quad \text { for all } t \in \mathcal{B}_{k}, 1 \leq k \leq m .
$$

Since $f_{e} \in P A P_{0}(\mathbb{Y}, \mathbb{X}, \rho)$, there exists $T_{0}>0$ such that

$$
\frac{1}{\mu(T, \rho)} \int_{-T}^{T}\left\|f_{e}\left(t, x_{k}\right)\right\| \rho(t) d t<\frac{\varepsilon}{2 m} \quad \text { for } T>T_{0}, 1 \leq k \leq m .
$$

Now by (3.5)-(3.7), for $T>T_{0}$,

$$
\begin{aligned}
& \frac{1}{\mu(T, \rho)} \int_{-T}^{T}\left\|f_{e}\left(t, h_{\mathrm{ap}}(t)\right)\right\| \rho(t) d t \\
& \leq \frac{1}{\mu(T, \rho)} \sum_{k=1}^{m} \int_{\mathcal{B}_{k} \bigcap[-T, T]}\left(\left\|f_{e}\left(t, h_{\mathrm{ap}}(t)\right)-f_{e}\left(t, x_{k}\right)\right\|+\left\|f_{e}\left(t, x_{k}\right)\right\|\right) \rho(t) d t \\
& \leq \frac{1}{\mu(T, \rho)} \sum_{k=1}^{m} \int_{\mathcal{B}_{k} \bigcap[-T, T]} \frac{\varepsilon \rho(t)}{2} d t \\
& \quad+\frac{1}{\mu(T, \rho)} \sum_{k=1}^{m} \int_{\mathcal{B}_{k} \cap[-T, T]}\left\|f_{e}\left(t, x_{k}\right)\right\| \rho(t) d t
\end{aligned}
$$




$$
\begin{aligned}
& =\frac{\varepsilon}{2 \mu(T, \rho)} \int_{-T}^{T} \rho(t) d t+\frac{1}{\mu(T, \rho)} \sum_{k=1}^{m} \int_{\mathcal{B}_{k} \bigcap[-T, T]}\left\|f_{e}\left(t, x_{k}\right)\right\| \rho(t) d t \\
& <\frac{\varepsilon}{2}+m \frac{\varepsilon}{2 m}=\varepsilon,
\end{aligned}
$$

which yields that $f_{e}\left(\cdot, h_{\mathrm{ap}}(\cdot)\right) \in P A P_{0}(\mathbb{X}, \rho)$. The proof is complete.

\section{REMARK 3.3.}

(i) Theorem 3.2 improves the composition theorem given in [10]. In fact, the following Lipschitz condition for $f \in P A P(\mathbb{Y}, \mathbb{X}, \rho), \rho \in U_{\infty}$, is necessary for the composition theorem in [10]:

$$
\|f(t, u)-f(t, v)\| \leq L\|u-v\| \quad \text { for all } u, v \in \mathbb{Y}, t \in \mathbb{R} .
$$

It is easy to verify that (3.8) implies the uniform continuity condition $\left(\mathrm{H}_{1}\right)$ and $\left(\mathrm{H}_{2}\right)$. On the other hand, Example 4.4 at the end of this paper shows that there are functions satisfying $\left(\mathrm{H}_{1}\right)$ and $\left(\mathrm{H}_{2}\right)$ but not satisfying (3.8).

(ii) In particular, if $\rho=1$ - that is, if the classical pseudo-almost periodic periodic functions are considered-Theorem 3.2 is the same as [12, Theorem 2.1].

\section{Semilinear abstract differential equations}

Consider the semilinear differential equations

$$
u^{\prime}(t)=A u(t)+f(t, u(t)), \quad t \in \mathbb{R},
$$

where $f \in P A P(\mathbb{X}, \mathbb{X}, \rho)$ with $\rho \in U_{T}$ and $A$ is the infinitesimal generator of an exponentially stable compact $C_{0}$-semigroup $\{T(t)\}_{t \geq 0}$ in $\mathbb{X}$, such that $\|T(t)\| \leq$ $M e^{-\omega t}$ for all $t \geq 0$ and some $M>0, \omega>0$.

The following lemma will be used in the proof of our main result in this section.

LEMMA 4.1. $P A P_{0}(\mathbb{X}, \rho)$ with $\rho \in U_{T}$ is translation invariant, that is, $\phi \in$ $\operatorname{PAP}_{0}(\mathbb{X}, \rho)$ and $s \in \mathbb{R}$ imply that $\phi(\cdot-s) \in \operatorname{PAP}_{0}(\mathbb{X}, \rho)$.

Proof. Let $\phi \in P A P_{0}(\mathbb{X}, \rho)$ and $s \in \mathbb{R}$. By [10, Theorem 3.3], $\phi \in P A P_{0}(\mathbb{X}, \rho)=$ $P A P_{0}\left(\mathbb{X}, \rho_{s}\right)$ since $\rho \in U_{T}$. Without loss of generality, we may assume that $s>0$. Meanwhile, $\rho \in U_{T}$ implies that $\rho \prec \rho_{2 s}$, that is, there exists $M>0$ such that $\rho_{2 s}(t) / \rho(t) \leq M$ for $t \in \mathbb{R}$. Then, for $T>s$,

$$
\begin{aligned}
\int_{-T-s}^{T+s} \rho_{s}(t) d t & =\int_{-T}^{T+2 s} \rho(t) d t=\mu(T, \rho)+\int_{T}^{T+2 s} \rho(t) d t \\
& =\mu(T, \rho)+\int_{T-2 s}^{T} \rho_{2 s}(t) d t \leq \mu(T, \rho)+\int_{-T}^{T} M \rho(t) d t \\
& =(M+1) \mu(T, \rho) .
\end{aligned}
$$


Therefore,

$$
\begin{aligned}
\frac{1}{\mu(T, \rho)} \int_{-T}^{T}\|\phi(t-s)\| \rho(t) d t & =\frac{1}{\mu(T, \rho)} \int_{-T-s}^{T-s}\|\phi(t)\| \rho_{s}(t) d t \\
& \leq \frac{1}{\mu(T, \rho)} \int_{-T-s}^{T+s}\|\phi(t)\| \rho_{s}(t) d t \\
& =\frac{\int_{-T-s}^{T+s} \rho_{s}(t) d t}{\mu(T, \rho) \int_{-T-s}^{T+s} \rho_{s}(t) d t} \cdot \int_{-T-s}^{T+s}\|\phi(t)\| \rho_{s}(t) d t \\
& \leq \frac{M+1}{\int_{-T-s}^{T+s} \rho_{s}(t) d t} \int_{-T-s}^{T+s}\|\phi(t)\| \rho_{s}(t) d t \\
& \rightarrow 0, \quad \text { as } T \rightarrow \infty
\end{aligned}
$$

which implies that $\phi(\cdot-s) \in P A P_{0}(\mathbb{X}, \rho)$. The proof is complete.

We recall that $u$ is said to be a mild weighted pseudo-almost periodic solution of (4.1) if $u \in P A P(\mathbb{X}, \rho)$, and

$$
u(t)=\int_{-\infty}^{t} T(t-s) f(s, u(s)) d s .
$$

Furthermore, $u$ is called a weighted pseudo-almost periodic solution of (4.1) if $u(t) \in D(A)$, for $t \in \mathbb{R}$, and is continuously differentiable in $t \in \mathbb{R}$ and satisfies (4.1).

To deal with the existence of mild weighted pseudo-almost periodic solutions of (4.1), we introduce the following assumptions on $f$ :

$\left(\mathrm{H}_{3}\right)$ There exists $L>0$ such that $\sup _{t \in \mathbb{R},\|u\| \leq L}\|f(t, u)\| \leq \omega L / M$.

$\left(\mathrm{H}_{4}\right)$ Let $\left\{u_{n}\right\} \subset P A P(\mathbb{X}, \rho)$ be uniformly bounded in $\mathbb{R}$ and uniformly convergent in each compact subset of $\mathbb{R}$. Then $\left\{f\left(\cdot, u_{n}(\cdot)\right)\right\}$ is relatively compact in $B C(\mathbb{R}, \mathbb{X})$.

We are now in a position to give the main result in this section.

TheOREM 4.2. Suppose that $f \in P A P(\mathbb{X}, \mathbb{X}, \rho)$ with $\rho \in U_{T}$ and $\left(\mathrm{H}_{1}\right)-\left(\mathrm{H}_{4}\right)$ are satisfied. Then (4.1) has a mild $\rho$-pseudo-almost periodic solution $u(t)$ such that $\|u\|=\sup _{t \in \mathbb{R}}\|u(t)\| \leq L$.

Proof. Let

$$
B=\{u \in P A P(\mathbb{X}, \rho):\|u\| \leq L\} .
$$

Clearly, $B$ is closed convex. Define $V: B C(\mathbb{R}, \mathbb{X}) \rightarrow B C(\mathbb{R}, \mathbb{X})$ by

$$
(V u)(t)=\int_{-\infty}^{t} T(t-s) f(s, u(s)) d s, \quad t \in \mathbb{R} .
$$

We only need to prove the existence of fixed points of $V$ in $B$, and this can be approached by Schauder's fixed point theorem. 
By assumption $\left(\mathrm{H}_{1}\right)$, it is easy to verify that $V$ is continuous. If we have

$$
V(B) \subset B,
$$

then by $\left(\mathrm{H}_{4}\right)$ and an argument similar to that in [12, Proof of Theorem 3.1], we can prove that $V$ has a fixed point in $\overline{c o} V B$ (here we omit the details). So it is sufficient to prove (4.2).

For $u \in B$ and $t \in \mathbb{R}$, by $\left(\mathrm{H}_{3}\right)$,

$$
\begin{aligned}
\|(V u)(t)\| & =\left\|\int_{-\infty}^{t} T(t-s) f(s, u(s)) d s\right\| \\
& \leq \int_{-\infty}^{t} M e^{-\omega(t-s)} \frac{\omega}{M} L d s=L,
\end{aligned}
$$

which shows that

$$
\|V u\| \leq L \quad \text { for } u \in B
$$

Let

$$
f=f_{\mathrm{ap}}+f_{e}, \quad u=u_{\mathrm{ap}}+u_{e} \in B,
$$

where $f_{\text {ap }} \in A P(\mathbb{X}, \mathbb{X}), f_{e} \in P A P_{0}(\mathbb{X}, \mathbb{X}, \rho), u_{\text {ap }} \in A P(\mathbb{X})$ and $u_{e} \in P A P_{0}(\mathbb{X}, \rho)$. It follows from Theorem 3.2 that $f(\cdot, u(\cdot)) \in P A P(\mathbb{X}, \mathbb{X}, \rho)$ with almost periodic component $f_{\text {ap }}\left(\cdot, u_{\text {ap }}(\cdot)\right)$. Then, for $\varepsilon>0$, there exists $\ell(\varepsilon)>0$ such that each interval of length $\ell(\varepsilon)>0$ contains a $\tau$ with the property that

$$
\left\|f_{\text {ap }}\left(t+\tau, u_{\text {ap }}(t+\tau)\right)-f_{\text {ap }}\left(t, u_{\text {ap }}(t)\right)\right\|<\frac{\omega \varepsilon}{M} \text { for } t \in \mathbb{R} .
$$

Decompose $V u$ as $V u=V_{1} u+V_{2} u$, where

$$
\begin{gathered}
\left(V_{1} u\right)(t)=\int_{-\infty}^{t} T(t-s) f_{\mathrm{ap}}\left(s, u_{\mathrm{ap}}(s)\right) d s, \\
\left(V_{2} u\right)(t)=\int_{-\infty}^{t} T(t-s)\left(f(s, u(s))-f_{\mathrm{ap}}\left(s, u_{\mathrm{ap}}(s)\right)\right) d s,
\end{gathered}
$$

for $t \in \mathbb{R}$. Noticing that $\|T(t)\| \leq M e^{-\omega t}$, by (4.4),

$$
\begin{aligned}
&\left\|\left(V_{1} u\right)(t+\tau)-\left(V_{1} u\right)(t)\right\| \\
&= \| \int_{-\infty}^{t} T(t-s) f_{\mathrm{ap}}\left(s+\tau, u_{\mathrm{ap}}(s+\tau)\right) d s \\
&-\int_{-\infty}^{t} T(t-s) f_{\mathrm{ap}}\left(s, u_{\mathrm{ap}}(s)\right) d s \| \\
& \leq \int_{-\infty}^{t}\|T(t-s)\| \cdot\left\|f_{\mathrm{ap}}\left(s+\tau, u_{\mathrm{ap}}(s+\tau)\right)-f_{\mathrm{ap}}\left(s, u_{\mathrm{ap}}(s)\right)\right\| d s \\
& \leq \int_{-\infty}^{t} M e^{-\omega(t-s)} \frac{\omega \varepsilon}{M} d s \\
&= \varepsilon
\end{aligned}
$$


for all $t \in \mathbb{R}$. This implies that

$$
V_{1} u \in A P(\mathbb{X}) \quad \text { for } u \in B
$$

On the other hand, it is clear that $f(\cdot, u(\cdot))-f_{\text {ap }}\left(\cdot, u_{\text {ap }}(\cdot)\right) \in B C(\mathbb{R}, \mathbb{X})$. Then

$$
\begin{aligned}
& \frac{1}{\mu(T, \rho)} \int_{-T}^{T}\left\|\left(V_{2} u\right)(t)\right\| \rho(t) d t \\
& \leq \frac{1}{\mu(T, \rho)} \int_{-T}^{T} \int_{-\infty}^{t}\|T(t-s)\| \cdot\left\|f(s, u(s))-f_{\mathrm{ap}}\left(s, u_{\mathrm{ap}}(s)\right)\right\| d s \rho(t) d t \\
& \leq \frac{M}{\mu(T, \rho)} \int_{-T}^{T} \int_{-\infty}^{t} e^{-\omega(t-s)}\left\|f(s, u(s))-f_{\mathrm{ap}}\left(s, u_{\mathrm{ap}}(s)\right)\right\| d s \rho(t) d t \\
&= \frac{M}{\mu(T, \rho)} \int_{-T}^{T} \int_{0}^{\infty} e^{-\omega s} \| f(t-s, u(t-s)) \\
&-f_{\mathrm{ap}}\left(t-s, u_{\mathrm{ap}}(t-s)\right) \| d s \rho(t) d t \\
&= M \int_{0}^{\infty} e^{-\omega s} \frac{1}{\mu(T, \rho)} \int_{-T}^{T} \| f(t-s, u(t-s)) \\
&-f_{\mathrm{ap}}\left(t-s, u_{\mathrm{ap}}(t-s)\right) \| \rho(t) d t d s \\
&= M \int_{0}^{\infty} e^{-\omega s} \Phi_{T}(s) d s,
\end{aligned}
$$

where

$$
\Phi_{T}(s)=\frac{1}{\mu(T, \rho)} \int_{-T}^{T}\left\|f(t-s, u(t-s))-f_{\mathrm{ap}}\left(t-s, u_{\mathrm{ap}}(t-s)\right)\right\| \rho(t) d t .
$$

Noticing that $f(\cdot, u(\cdot))-f_{\text {ap }}\left(\cdot, u_{\text {ap }}(\cdot)\right) \in P A P_{0}(\mathbb{X}, \rho)$, then by Lemma 4.1 , for each $s \in \mathbb{R}$, it is easy to get that

$$
\lim _{T \rightarrow \infty} \Phi_{T}(s) \rightarrow 0
$$

and

$$
\left\|\Phi_{T}(s)\right\| \leq\left\|f(\cdot, u(\cdot))-f_{\text {ap }}\left(\cdot, u_{\text {ap }}(\cdot)\right)\right\| .
$$

Now, by the Lebesgue dominated convergence theorem,

$$
\lim _{T \rightarrow \infty} \frac{1}{\mu(T, \rho)} \int_{-T}^{T}\left\|\left(V_{2} u\right)(t)\right\| \rho(t) d t \leq \lim _{T \rightarrow \infty} M \int_{0}^{\infty} e^{-\omega s} \Phi_{T}(s) d s=0,
$$

which implies that $V_{2} u \in P A P_{0}(\mathbb{X}, \rho)$, and then by (4.5) we have $V u \in P A P(\mathbb{X}, \rho)$. This together with (4.3) leads to (4.2). The proof is complete.

For the $\rho$-pseudo-almost periodic solution of (4.1), we have the following result. 
THEOREM 4.3. Let $u(t)$ be a mild $\rho$-pseudo-almost periodic solution of (4.1), and suppose that

$$
f(t, u(t)) \in D(A) \text { for } t \in \mathbb{R}, \quad A f(\cdot, u(\cdot)) \in L^{1}(\mathbb{R}, \mathbb{X}) .
$$

Then $u(t)$ is a $\rho$-pseudo-almost periodic solution of (4.1).

PROOF. Since $u(t)$ is a mild $\rho$-pseudo-almost periodic solution of (4.1),

$$
u(t)=\int_{-\infty}^{t} T(t-s) f(s, u(s)) d s .
$$

By (4.6),

$$
\begin{aligned}
\frac{d_{+} u(t)}{d t}= & \lim _{h \rightarrow 0+} \frac{1}{h}(u(t+h)-u(t)) \\
= & \lim _{h \rightarrow 0+}\left[\int_{-\infty}^{t} \frac{1}{h}(T(t+h-s)-T(t-s)) f(s, u(s)) d s\right. \\
& \left.\quad+\frac{1}{h} \int_{t}^{t+h} T(t+h-s) f(s, u(s)) d s\right] \\
= & \int_{-\infty}^{t} T(t-s) A f(s, u(s)) d s+f(t, u(t)) .
\end{aligned}
$$

This shows that $d_{+} u(t) / d t$ is continuous, and then $u(t)$ is continuously differentiable in $t \in \mathbb{R}$. Noticing that $A$ is closed,

$$
u^{\prime}(t)=A u(t)+f(t, u(t)) .
$$

That is, $u(t)$ is a $\rho$-pseudo-almost periodic solution. The proof is complete.

To conclude this paper, we give an example involving the heat equation.

EXAMPLE 4.4. Consider the heat equation given by the system

$$
\left\{\begin{array}{l}
\frac{\partial v(t, x)}{\partial t}=\frac{\partial^{2} v(t, x)}{\partial x^{2}}+\sin t+\sin (\pi t)+a(t) g(t, v(t, x)) \quad \text { for } t \in \mathbb{R}, x \in[0,1], \\
v(t, 0)=v(t, 1)=0 \quad \text { for } t \in \mathbb{R}
\end{array}\right.
$$

where $a(t)=\min \left\{1, e^{-t}\right\}$ and

$$
g(t, v(t, x))= \begin{cases}\sin \frac{v(t, x)}{1+t^{2}} \sin \frac{1}{v(t, x)} & \text { for } v(t, x) \neq 0 \\ 0 & \text { for } v(t, x)=0 .\end{cases}
$$

Let $\mathbb{X}=L^{2}[0,1]$, and define $A: \mathbb{X} \rightarrow \mathbb{X}$ by $A u(\cdot)=u^{\prime \prime}(\cdot)$ with domain

$$
D(A)=\left\{u(\cdot) \in \mathbb{X}: u^{\prime \prime}(\cdot) \in \mathbb{X}, u(0)=u(1)=0\right\} .
$$


It is well known that $A$ is the infinitesimal generator of a compact $C_{0}$-semigroup $\{T(t)\}_{t \geq 0}$ satisfying

$$
\|T(t)\| \leq e^{-t} \quad \text { for } t \geq 0 .
$$

Then Equation (4.7) can be formulated by the following abstract equation:

$$
u^{\prime}(t)=A u(t)+f(t, u(t)), \quad t \in \mathbb{R},
$$

where $u(t)=v(t, \cdot)$ and $f: \mathbb{R} \times \mathbb{X} \rightarrow \mathbb{X}$ with

$$
f(t, u(t))=\sin t+\sin (\pi t)+a(t) g(t, u(t))
$$

for all $(t, u(t)) \in \mathbb{R} \times \mathbb{X}$.

It is not hard to verify that $e^{t} \in U_{T}, f \in P A P\left(\mathbb{X}, \mathbb{X}, e^{t}\right)$ and $f$ satisfies $\left(\mathrm{H}_{1}\right)-\left(\mathrm{H}_{4}\right)$ with $L=3$. Then by Theorem 4.2, (4.8) has a mild weighted pseudo-almost periodic solution $u \in P A P\left(\mathbb{X}, e^{t}\right)$ such that

$$
\|u\|=\sup _{t \in \mathbb{R}}\|u(t)\|_{L^{2}[0,1]} \leq 3 .
$$

That is, system (4.7) has a mild weighted pseudo-almost periodic solution $v(t, x)$ such that $u(t)=v(t, \cdot), t \in \mathbb{R}, u \in P A P\left(\mathbb{X}, e^{t}\right)$ and

$$
\|u\|=\sup _{t \in \mathbb{R}}\|v(t, \cdot)\|_{L^{2}[0,1]}=\sup _{t \in \mathbb{R}}\left(\int_{0}^{1}|v(t, x)|^{2} d x\right)^{1 / 2} \leq 3 .
$$

However, it is obvious that $f$ is not Lipschitz continuous. As a result, [10, Theorem 4.2] is not applicable.

\section{Acknowledgement}

The authors are grateful to the referee for valuable comments and corrections.

\section{References}

[1] E. Ait Dads and O. Arino, 'Exponential dichotomy and existence of pseudo almost-periodic solutions for some differential equations', Nonlinear Anal. 27 (1996), 369-386.

[2] E. Ait Dads, P. Cieutat and K. Ezzinbi, 'The existence of pseudo almost-periodic solutions for some nonlinear differential equations in Banach spaces', Nonlinear Anal. 69 (2008), 1325-1342.

[3] E. Ait Dads, K. Ezzinbi and O. Arino, 'Pseudo almost-periodic solutions for some differential equations', Nonlinear Anal. 28 (1997), 1141-1155.

[4] B. Amir and L. Maniar, 'Composition of pseudo almost-periodic functions and Cauchy problems with operator of nondense domain', Ann. Math. Blaise Pascal 6 (1999), 1-11.

[5] C. Corduneanu, Almost Periodic Functions, 2nd edn (Chelsea, New York, 1989).

[6] C. Cuevas and C. Lizama, 'Almost automorphic solutions to a class of semilinear fractional differential equations', Appl. Math. Lett. 21 (2008), 1315-1319.

[7] C. Cuevas and M. Pinto, 'Existence and uniqueness of pseudo-almost periodic solutions of semilinear Cauchy problem with non dense domain', Nonlinear Anal. 45 (2001), 73-83.

[8] T. Diagana, 'Weighted pseudo almost periodic functions and applications', C. R. Acad. Sci. Paris, Ser. I 343 (2006), 643-646. 
[9] T. Diagana, 'Existence and uniqueness of pseudo almost periodic solutions to some classes of partial evolution equations', Nonlinear Anal. 66 (2007), 384-395.

[10] T. Diagana, 'Weighted pseudo-almost periodic solutions to some differential equations', Nonlinear Anal. 68 (2008), 2250-2260.

[11] A. M. Fink, Almost Periodic Differential Equations, Lecture Notes in Mathematics, 377 (Springer, New York, 1974).

[12] H. X. Li, F. L. Huang and J. Y. Li, 'Composition of pseudo almost-periodic functions and semilinear differential equations', J. Math. Anal. Appl. 255 (2001), 436-446.

[13] G. M. N'Guérékata, Almost Automorphic Functions and Almost Periodic Functions in Abstract Spaces (Kluwer Academic/Plenum Publishers, New York, London, Moscow, 2001).

[14] C. Y. Zhang, 'Pseudo almost periodic solutions of some differential equations', J. Math. Anal. Appl. 181 (1994), 62-76.

[15] C. Y. Zhang, 'Integration of vector-valued pseudo almost periodic functions', Proc. Amer. Math. Soc. 121 (1994), 167-174.

[16] C. Y. Zhang, 'Pseudo almost periodic solutions of some differential equations, II', J. Math. Anal. Appl. 192 (1995), 543-561.

\section{LI-LI ZHANG, Department of Mathematics, Sichuan University, Chengdu, Sichuan 610064, PR China \\ e-mail: zllyou@126.com}

HONG-XU LI, Department of Mathematics, Sichuan University, Chengdu, Sichuan 610064, PR China

e-mail: hoxuli@sohu.com 